\title{
ADGRG1 enriches for functional human hematopoietic stem cells following ex vivo expansion-induced mitochondrial oxidative stress
}

\author{
Yandan Chen, ${ }^{1}$ Shuyi Fang, ${ }^{2}$ Qingwei Ding, ${ }^{3}$ Rongzhen Jiang, ${ }^{4}$ Jiefeng He, ${ }^{5}$ Qin Wang, ${ }^{6}$ Yuting Jin, ${ }^{1,4}$ Xinxin Huang, ${ }^{7}$ Sheng Liu, ${ }^{8}$ \\ Maegan L. Capitano, ${ }^{9}$ Thao Trinh, ${ }^{9}$ Yincheng Teng, ${ }^{4}$ Qingyou Meng, ${ }^{3}$ Jun Wan, ${ }^{2,8,10}$ Hal E. Broxmeyer, ${ }^{9}$ and Bin Guo ${ }^{1}$ \\ 'Department of Pathophysiology, Key Laboratory of Cell Differentiation and Apoptosis of the Chinese Ministry of Education, Shanghai Jiao Tong University School of Medicine, Shanghai, China. ${ }^{2}$ Department \\ of BioHealth Informatics, Indiana University School of Informatics and Computing at Indiana University-Purdue University Indianapolis (IUPUI), Indianapolis, Indiana, USA. ${ }^{3}$ Department of Vascular \\ Surgery, General Surgery Clinical Center, Shanghai General Hospital, Shanghai Jiao Tong University School of Medicine, Shanghai, China. ${ }^{4}$ Obstetrics Intensive Care Center, The Institute of Obstetrics and \\ Gynecology, Department of Obstetrics and Gynecology, Affiliated Sixth People's Hospital, Shanghai Jiao Tong University, Shanghai, China. ${ }^{5}$ Department of General Surgery, Shanxi Bethune Hospital, Shanxi \\ Medical University, Taiyuan, Shanxi, China. ${ }^{6}$ Department of Gynaecology and Obstetrics, the First People's Hospital of Kunshan, Kunshan, China. ${ }^{7}$ Xuhui Hospital and Institutes of Biomedical Sciences, Fudan \\ University, Shanghai, China. ${ }^{8}$ Department of Medical and Molecular Genetics, ${ }^{9}$ Department of Microbiology and Immunology, and ${ }^{10}$ Center for Computational Biology and Bioinformatics, Indiana University \\ School of Medicine, Indianapolis, Indiana, USA.
}

\begin{abstract}
The heterogeneity of human hematopoietic stem cells (HSCs) and hematopoietic progenitor cells (HPCs) under stress conditions such as ex vivo expansion is poorly understood. Here, we report that the frequencies of SCID-repopulating cells were greatly decreased in cord blood (CB) CD34+ HSCs and HPCs upon ex vivo culturing. Transcriptomic analysis and metabolic profiling demonstrated that mitochondrial oxidative stress of human CB HSCs and HPCs notably increased, along with loss of stemness. Limiting dilution analysis revealed that functional human HSCs were enriched in cell populations with low levels of mitochondrial ROS (mitoROS) during ex vivo culturing. Using single-cell RNA-Seq analysis of the mitoROS low cell population, we demonstrated that functional HSCs were substantially enriched in the adhesion GPCR G1-positive (ADCRG1+) population of $\mathrm{CD} 34{ }^{+} \mathrm{CD} 133^{+} \mathrm{CB}$ cells upon ex vivo expansion stress. Gene set enrichment analysis revealed that HSC signature genes including MSI2 and MLLT3 were enriched in CD34+CD133+ADGRG1+ CB HSCs. Our study reveals that ADCRG1 enriches for functional human HSCs under oxidative stress during ex vivo culturing, which can be a reliable target for drug screening of agonists of HSC expansion.
\end{abstract}

\section{Introduction}

Hematopoietic stem cell transplantation (HSCT) is a well-established and effective stem cell-based therapy and is used to treat many hematological and nonhematological diseases (1-3). However, transplantations that are performed with limited numbers of HSCs, as with CB or gene-edited HSCs, may cause incomplete hematopoietic reconstitution or hematopoietic failure (4). Ex vivo expansion is one potential means to overcome the limitations of the rarity of HSCs (5-8). Given the extensive loss of stemness upon ex vivo culturing of HSCs, clinically applicable ex vivo expansion systems have not yet been developed for extensive clinical use $(8,9)$. The inconsistency between phenotyping and function of human HSCs after ex vivo culturing has been reported (10). Deciphering mechanisms and the regulation of human HSC selfrenewal, especially under stress conditions such as ex vivo culturing, would facilitate the development of ex vivo expansion systems

Authorship note: YC, SF, QD, and RJ contributed equally to this work. Conflict of interest: The authors have declared that no conflict of interest exists. Copyright: (๖) 2021, American Society for Clinical Investigation.

Submitted: February 3, 2021; Accepted: August 24, 2021; Published: October 15, 2021. Reference information: J Clin Invest. 2021;131(20):e148329.

https://doi.org/10.1172/JCl148329. of functional HSCs for clinical use and improve our understanding of the regulation of stem cell biology, which is still in its infancy.

\section{Results and Discussion}

Functional CB HSCs are enriched in mitochondrial ROS low CD $34^{+}$ cell populations upon ex vivo culture-induced mitochondrial oxidative stress. To explore molecular mechanisms of human HSC maintenance under stress conditions, we first compared the reconstituting capacity of freshly isolated or ex vivo-cultured human cord blood (CB) $\mathrm{CD}_{3} 4^{+}$HSCs and hematopoietic progenitor cells (HPCs) by transplanting the exact same number of $\mathrm{CD} 4^{+}$cells into sublethally irradiated immune-compromised NOD-scid IL2Rg null (NSG) recipient mice. SCID repopulating cells (SRCs) in day-4-cultured $\mathrm{CB}$ CD $34^{+}$cells and freshly isolated $\mathrm{CB} \mathrm{CD} 34^{+}$cells were assessed by limiting dilution analysis (LDA) (1). Poisson distribution analysis revealed an SRC frequency of 1:3424 in uncultured day-O CB CD34 ${ }^{+}$cells and 1:27,194 in day-4 ex vivo-cultured CB CD $34^{+}$cells, suggesting the presence of, respectively, 92 SRCs and 37 SRCs (7.9-fold decrease upon ex vivo culturing using stem cell factor [SCF], thrombopoietin [TPO], and Flt3L in serum-free expansion medium [SFEM]) in $1 \times$ $10^{6}$ freshly isolated and ex vivo-cultured cells, respectively (Figure 1, A and B, Supplemental Figure 1A, and Supplemental Tables 
A

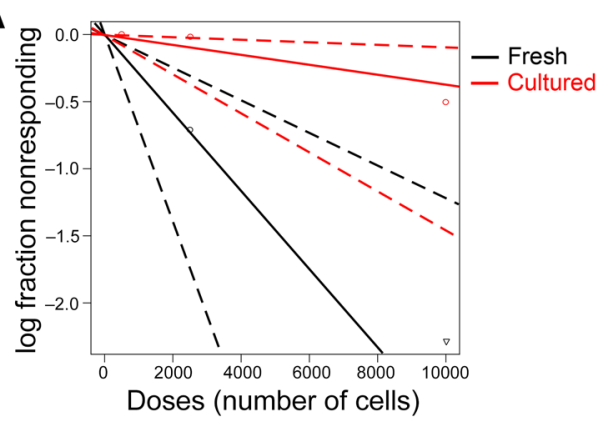

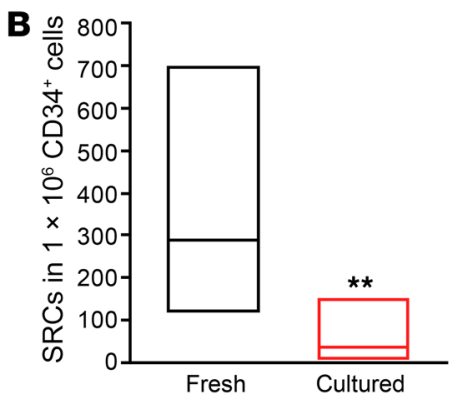

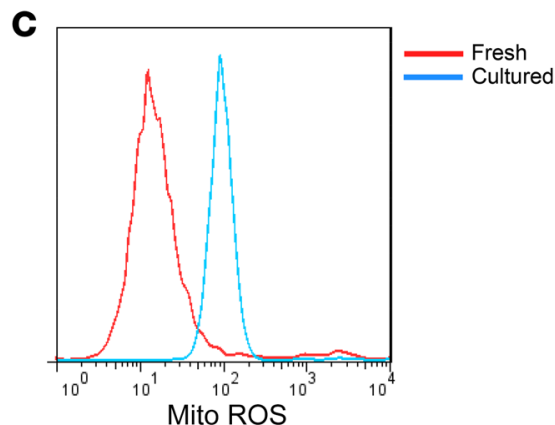

D

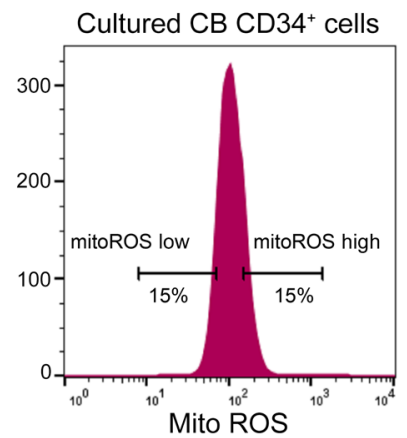

MitoROS low

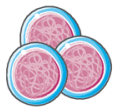

$\mathbf{E}$

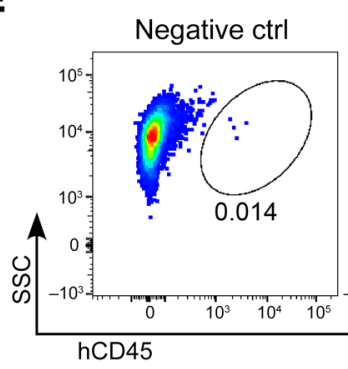

G

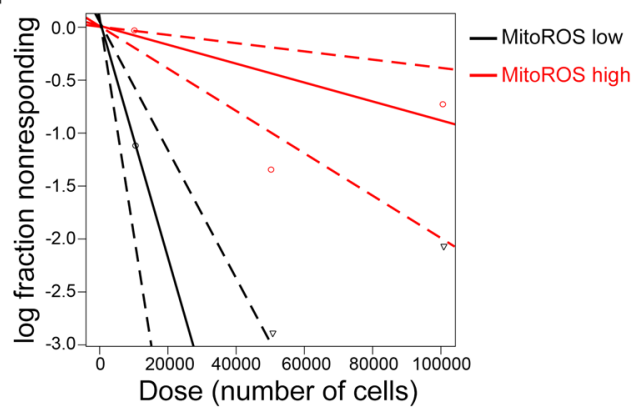

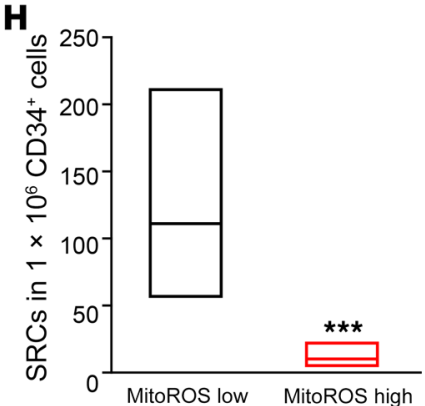

SRC determination by LDA

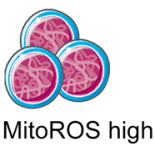

NSG

$\mathbf{F}$
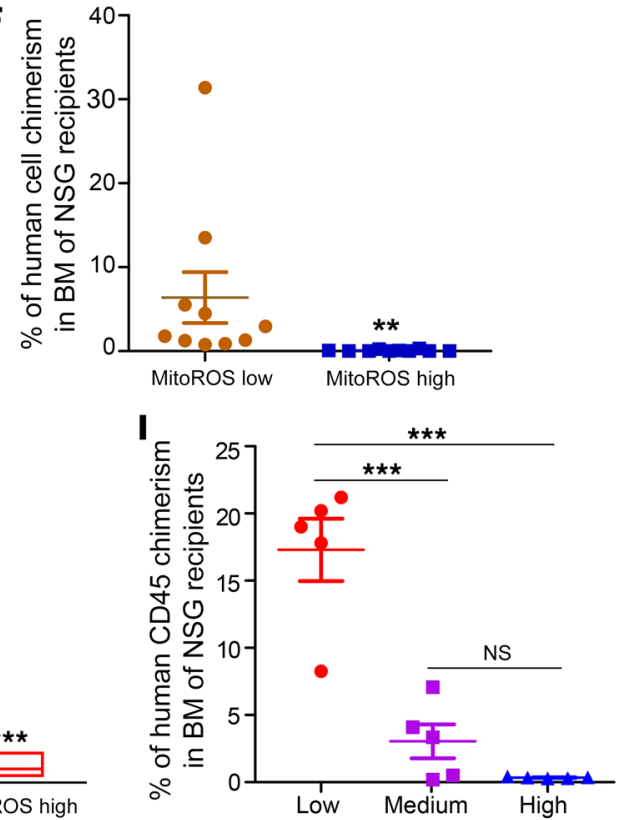

Figure 1. Functional CB HSCs are enriched in mitoROS low CD34+ cells upon ex vivo culture-induced mitochondrial oxidative stress. (A and B) Frequency of human SRCs in freshly isolated CB CD34+ cells or ex vivo-cultured CB CD34+ cells, as determined by LDA. $n=4-5$ mice per group. ${ }^{* *} P<0.01$; Poisson statistical analysis. (C) Histogram of mitoROS levels of fresh human CB CD34+ cells or cultured CB CD34+ cells. Representative histograms from 4 independent experiments are shown. (D) Strategy for SRC determination by LDA in mitoROS low CB CD34+ cells or mitoROS high CB CD34+ cells upon ex vivo culturing. (E and F) Percentage of hCD45 ${ }^{+}$cell chimerism in the BM of NSG mice 16 weeks after transplantation with 10,000 mitoROS low CB CD34 ${ }^{+}$cells or mitoROS high CB CD34+ cells upon ex vivo culturing. $n=10$ mice per group. (F) Statistical data are shown as dot plots (mean $\pm \mathrm{SEM})$. ${ }^{* *} P<0.01$, by 2 -tailed Student's $t$ test. ( $\mathbf{G}$ and $\mathbf{H}$ ) Frequency of human SRCs in mitoROS low CB CD34+ cells or mitoROS high CB CD34+ cells upon ex vivo culture stress. $n=4-15$ mice per group. ${ }^{* *} P<0.001$; Poisson statistical analysis. (I) Percentage of $h C D 45^{+}$cell chimerism in the BM of NSG mice 4 months after transplantation with 50,000 mitoROS low CB CD34+ cells, mitoROS medium CB CD34+ cells, or mitoROS high CB CD34+ cells following ex vivo culturing. Representative data from 2 independent experiments are shown. $n=5$ mice per group. ${ }^{* *} P<0.001$, by 1 -way ANOVA. NS, nonsignificant.

1 and 2; supplemental material available online with this article; https://doi.org/10.1172/JCI148329DS1). Human cell chimerism of the fresh $\mathrm{CB} C D 34^{+}$cell-infused group was significantly increased compared with that of the ex vivo-cultured $\mathrm{CB} \mathrm{CD} 34^{+}$ cell-infused group in both primary and secondary recipient mice (Supplemental Figure 1, B-E). These data demonstrate that the stemness of human CB HSCs and HPCs decreased upon ex vivo culturing with cytokines. 
A
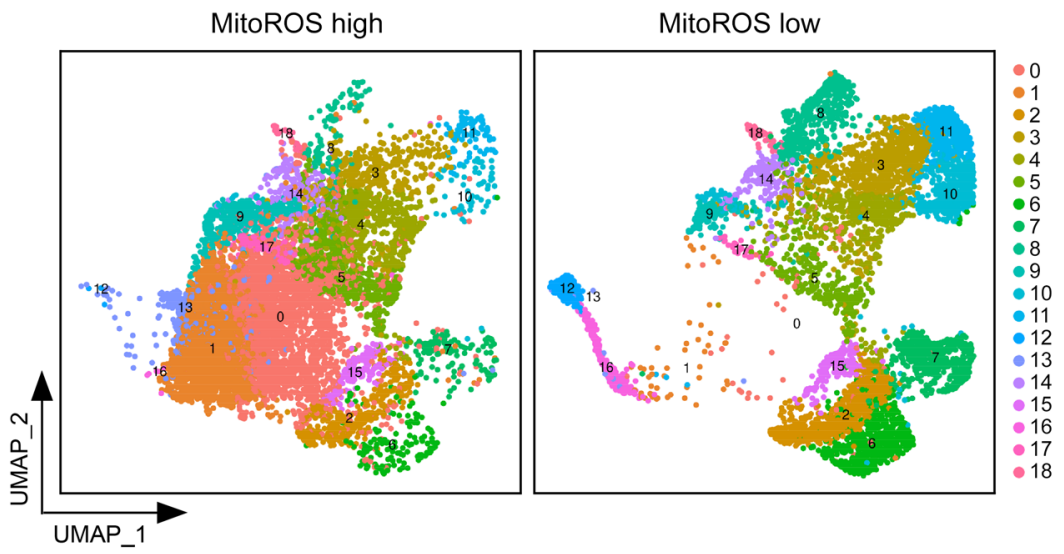

D

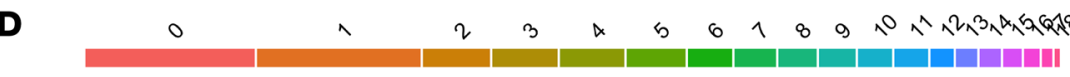

B

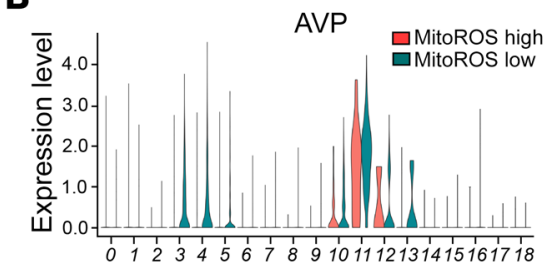

C

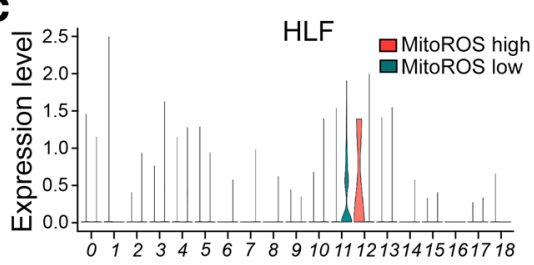

E

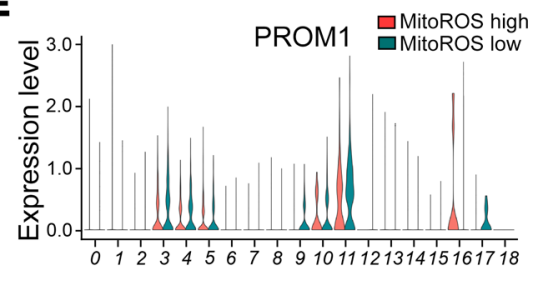

$\mathbf{F}$

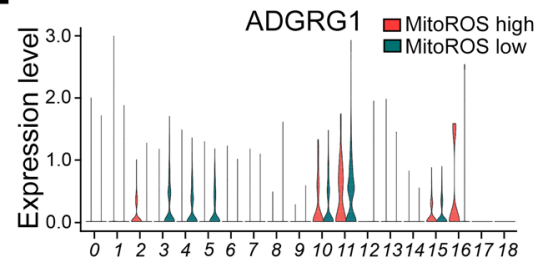

G

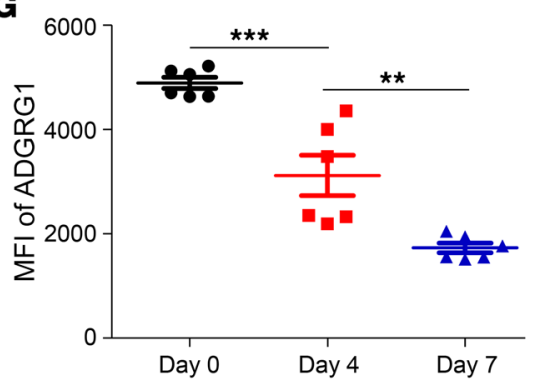

J

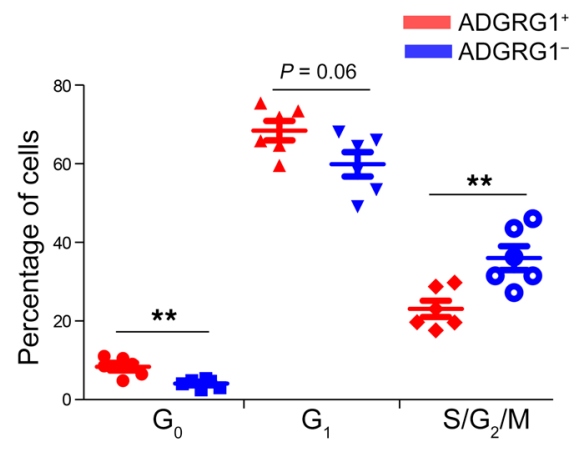


Figure 2. scRNA-Seq identifies ADGRG1 as a marker of functional CB HSCs upon mitochondrial oxidative stress induced by ex vivo culturing. (A) mitoROS high and mitoROS low CB CD34+ cells upon ex vivo culturing were analyzed by scRNA-Seq. (B and $\mathbf{C}$ ) Violin plots show the expression of HSC signature genes (AVP and HLF) in different clusters. (D) Heatmap of differentially expressed genes in 18 cell clusters of mitoROS low CB CD34 cells upon oxidative stress. (E and $\mathbf{F}$ ) Violin plots show the expression of CD133 (PROM1) and ADCRG1 in different clusters of both mitoROS low and mitoROS high $\mathrm{CB} C \mathrm{CD} 34^{+}$cells upon oxidative stress. (C) Dot plots showing the MFI of surface ADGRG1 in day-0, day-4, and day-7 ex vivo-cultured CB CD34 ${ }^{+}$cells. ${ }^{* *} P<0.01$ and ${ }^{* *} P<0.001$, by 1 -way ANOVA. (H) Representative flow histogram showing ADGRG1 expression in mitoROS low and mitoROS high CD34+ ${ }^{+}$cells. (I) Representative flow histogram showing mitoROS level in ADGRG1+CD34+CD133+ cells and ADCRG1-CD34+CD133 cells. (J) Quantification data showing the percentage of $G_{0}-, G_{-}^{-}$, and $\mathrm{S} / \mathrm{C}_{2} / \mathrm{M}$-stage cells in ADGRG1+CD34+CD133+ and ADCRG1-CD34+CD133+ cell populations. ${ }^{* *} P<0.01$, by 1 -way ANOVA. SSC, side scatter.

We performed RNA-Seq analysis to compare differentially expressed genes between freshly isolated and ex vivo-cultured CD $34^{+}$HSCs and HPCs with different engraftment capacities. Gene set enrichment analysis (GSEA) and gene ontology (GO) analysis suggested that many mitochondrion-related genes were apparently highly expressed in ex vivo-cultured $\mathrm{CB}$ CD $34^{+}$cells rather than freshly isolated $\mathrm{CB}$ CD $34^{+}$cells (Supplemental Figure 2 , A and B). We measured the extracellular oxygen consumption rate (OCR) with a Seahorse XF extracellular flux instrument. Consistent with the RNA-Seq results, we observed that the OCR of ex vivo-cultured $\mathrm{CB} C D 34^{+}$cells was significantly higher than the OCR of freshly isolated CB CD $34^{+}$cells (Supplemental Figure 2C). Mitochondrial ROS (mitoROS), mitochondrial mass, and mitochondrial membrane potential all markedly increased after ex vivo culturing (Figure $1 \mathrm{C}$ and Supplemental Figure 2, D-I), demonstrating that mitochondrial oxidative stress in CB HSCs and HPCs was induced by ex vivo expansion.

We then sought to determine whether human CB HSCs could be enriched in $\mathrm{CD}_{3} 4^{+}$cells with low levels of mitoROS upon ex vivo culture stress. We sorted $\mathrm{CD} 34^{+}$cells with low levels of mitoROS (mitoROS low, 15\%) and CD34+ cells with high levels of mitoROS (mitoROS high, 15\%) after 4 days of culturing, and then transplanted these 2 cell populations into sublethally irradiated NSG mice (Figure 1D). The human cell chimerism in the mitoROS low group was significantly higher than that in the mitoROS high group (Figure 1, E and F). Poisson distribution analysis revealed an SRC frequency of 1:8918 in the mitoROS low CB CD34 ${ }^{+}$cells and 1:112,939 in the mitoROS high $\mathrm{CB}$ CD34 $4^{+}$cells, suggesting the presence of 112 SRCs and 9 SRCs (12.4-fold decrease) in $1 \times 10^{6}$ mitoROS low versus mitoROS high ex vivo-cultured CD $34^{+}$cells, respectively (Figure 1, G and H, and Supplemental Tables 3 and 4). We also compared the engraftment capacity of mitoROS low, mitoROS high, and mitoROS medium (the remaining $70 \%$ of cells that were not mitoROS low or mitoROS high) $\mathrm{CD} 34^{+}$cells after ex vivo culturing. Human CD45 (hCD45) cell chimerism in the mitoROS low group was significantly higher than that in the mitoROS medium group (5.7-fold increase; Figure 1I and Supplemental Figure 2J). These results demonstrate that functional HSCs were mainly enriched in mitoROS low $\mathrm{CD}_{3} 4^{+}$cells under mitochondrial oxidative stress conditions due to ex vivo culturing.
Single-cell RNA-Seq analysis reveals that adhesion GPCR G1 marks functional human CB HSCs upon expansion. Single-cell RNASeq (scRNA-Seq) analysis is an established approach to study cell heterogeneity and characterize different cell subsets $(11,12)$. Next, we performed parallel transcriptomic analysis of mitoROS low and mitoROS high $\mathrm{CD} 34^{+}$cells at a single-cell level to identify HSC populations in $\mathrm{CD} 34^{+}$cells upon oxidative stress by ex vivo culturing. A total of 7663 mitoROS low $\mathrm{CD} 4^{+}$cells and 12,688 mitoROS high $\mathrm{CD}_{3} 4^{+}$cells were effectively sequenced and characterized, respectively (Supplemental Figure 3, A-D, and Supplemental Figure 4, A and B). We identified a total of 19 distinct cell clusters as shown in the uniform manifold approximation and projection (UMAP). Among them, populations of clusters 10, 11, 12, and 16 dramatically increased in mitoROS low CD34+ cells (Figure 2A). Established human HSC signature genes including arginine vasopressin (AVP) and hepatic leukemia factor (HLF) were substantially enriched in cluster 11, particularly for mitoROS low $\mathrm{CD}^{+} 4^{+}$cells (Figure 2, B and C). Moreover, many HSC functional regulators (13-17) such as aldehyde dehydrogenase 1 family member A1 (ALDH1A1), mixed-lineage leukemia translocated to chromosome 3 (MLLT3), musashi RNA-binding protein 2 (MSI2), and thioredoxin-interacting protein (TXNIP) were highly expressed in cluster 11 of the mitoROS low CD34+ cells (Figure 2D), suggesting that cluster 11 of mitoROS low $\mathrm{CD} 34^{+}$cells upon ex vivo culturing might be the more functional subset of HSCs.

We then checked whether cluster 11 could be colabeled by any cell-surface markers. Protein-protein interaction (PPI) network analysis demonstrated that adhesion GPCR G1 (ADGRG1) was associated with HSC surface markers including CD34 and CD133 (PROM1) (Supplemental Figure 4C). ADGRG1 was specifically expressed in cluster 11 cells, which were enriched in the CD34 ${ }^{+}$ $\mathrm{CD}_{133}{ }^{+} \mathrm{ADGRG} 1^{+}$population (Figure 2, E and F).

Approximately $90 \%$ of day-0 CB CD $34^{+}$cells were ADGRG1 $1^{+}$, and the percentages of $\mathrm{ADGRG} 1^{+}$cell populations in day- 4 and day-7 ex vivo-cultured $\mathrm{CB}$ CD $34^{+}$cells were, respectively, approximately $30 \%$ and $25 \%$ (Supplemental Figure $5 \mathrm{~A}$ ). We found that ADGRG1 was highly expressed in freshly isolated CB HSCs, multipotent progenitors, and $\mathrm{CD} 34^{+} \mathrm{CD} 38^{-}$cells, whereas its expression was relatively lower in $\mathrm{CD} 34^{+} \mathrm{CD} 38^{+}$and $\mathrm{CD} 34^{-} \mathrm{CB}$ cells (Supplemental Figure 5B). Surface expression of ADGRG1 was significantly decreased in day-4 and day-7 ex vivo-cultured $\mathrm{CD} 34^{+} \mathrm{CD} 133^{+}$ cells compared with that of day- $0 \mathrm{CD} 34^{+} \mathrm{CD} 133^{+}$cells (Figure $2 \mathrm{G}$ and Supplemental Figure $5 \mathrm{C}$ ). After ex vivo culturing, we found that ADGRG1 expression was notably higher in mitoROS low $\mathrm{CD}^{+} 4^{+}$cells than in mitoROS high $\mathrm{CD}^{+} 4^{+}$cells (Figure $2 \mathrm{H}$ ). Meanwhile, $\mathrm{CD} 34^{+} \mathrm{CD} 133^{+} \mathrm{ADGRG} 1^{+}$cells had significantly lower levels of mitoROS compared with $\mathrm{CD} 34^{+} \mathrm{CD} 133^{+} \mathrm{ADGRG} 1^{-}$cells (Figure 2I and Supplemental Figure 5D), and OCR analysis indicated that mitochondrial respiration activity was lower in $\mathrm{CD} 34^{+}$ $\mathrm{CD}_{133}{ }^{+} \mathrm{ADGRG} 1^{+}$cells (Supplemental Figure 5E). The percentage of $\mathrm{G}_{0}$-stage cells was notably higher in $\mathrm{CD} 34^{+} \mathrm{CD} 133^{+} \mathrm{ADGRG} 1^{+}$ cells than the that in $\mathrm{CD} 34^{+} \mathrm{CD} 133^{+} \mathrm{ADGRG} 1^{-}$cells (Figure $2 \mathrm{~J}$ and Supplemental Figure 5F). Our data indicate that ADGRG1 might be used for the enrichment of functional HSCs upon expansion.

ADGRG1 enriches for functional human CB HSCs upon expansion-induced oxidative stress. We performed long-term transplantation experiments using 3 different cell doses to determine whether 
A

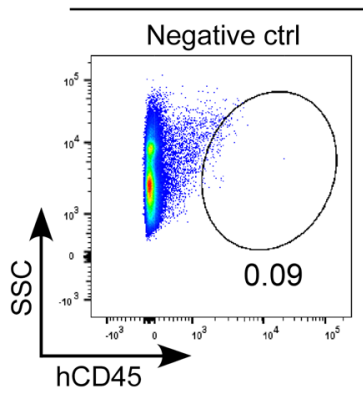

Primary transplantation

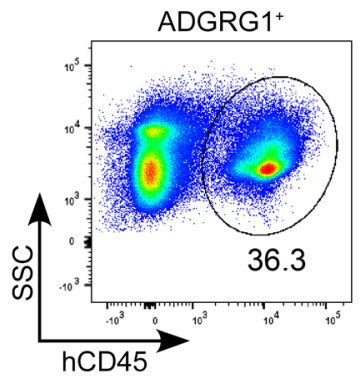

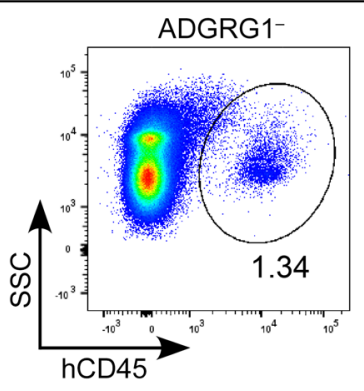

D

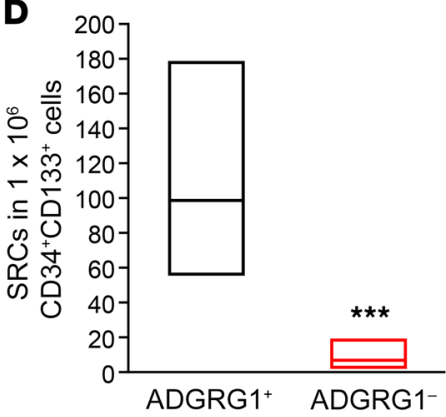

B

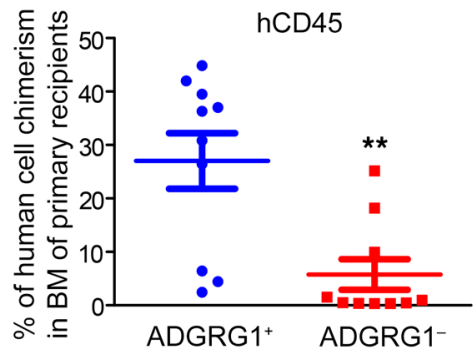

E

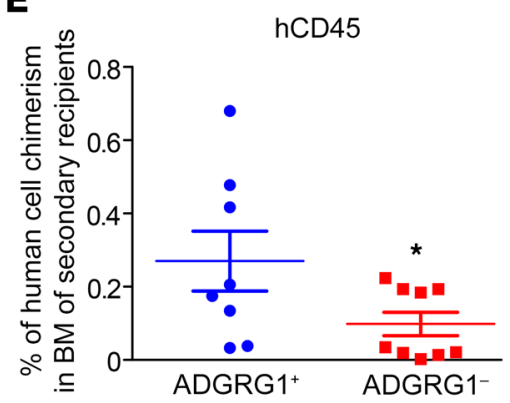

$\mathbf{F}$

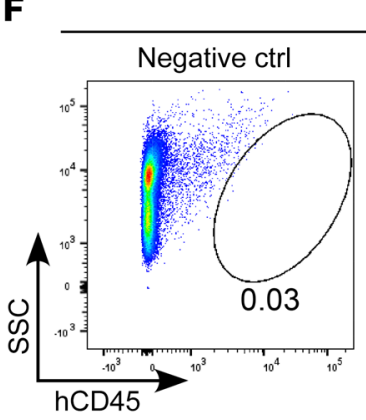

Secondary transplantation
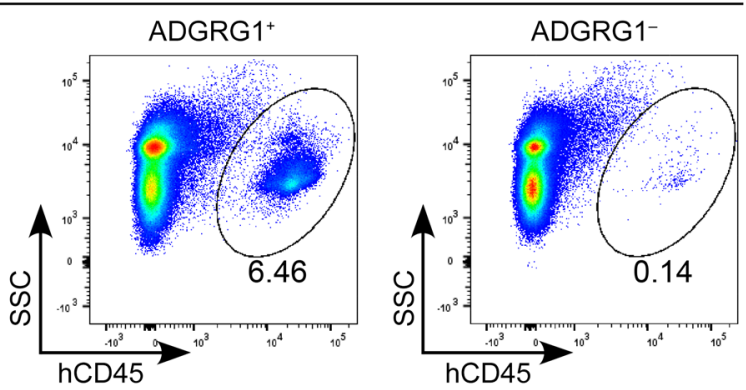

G

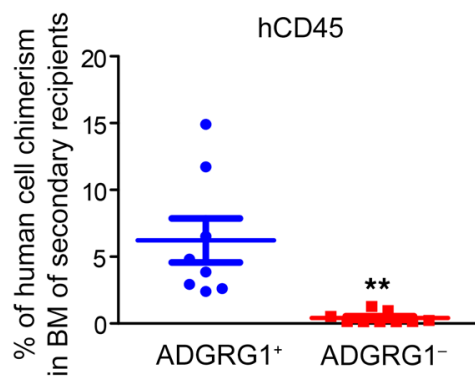

H

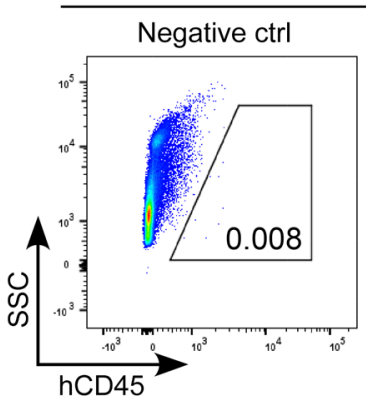

Homing

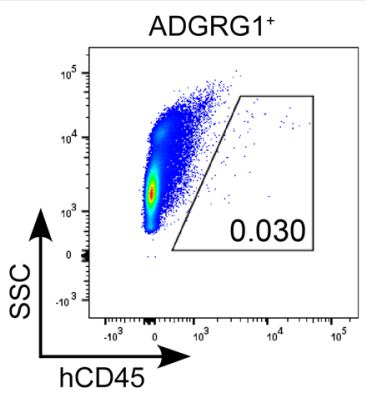

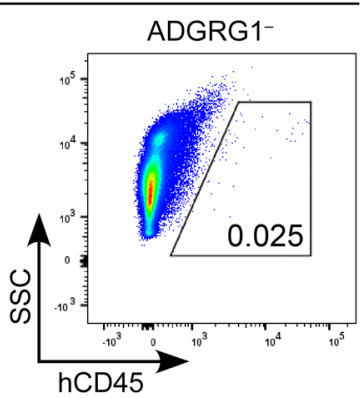

I

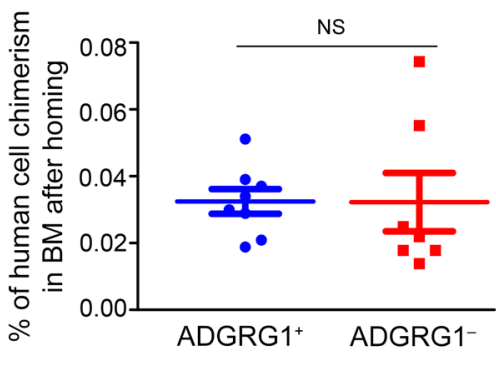

Figure 3. ADCRG1 enriches for functional CB HSCs upon mitochondrial oxidative stress induced by ex vivo culturing. (A and B) The percentage of hCD45 cells in BM of NSG recipients transplanted with 50,000 CD34+CD133+ADGRG1+ CB cells or CD34+CD133+ADCRG1- CB cells was determined by FACS. Quantification data are shown as dot plots (mean \pm SEM) in B. $n=10$ mice per group. ${ }^{* *} P<0.01$, by 2 -tailed Student's $t$ test. Ctrl, control. (C and D) Human SRCs in ADGRG1+ and ADGRG- CB CD34+CD133+ cells upon ex vivo culturing, as determined by LDA. $n=10$ mice per group. ${ }^{* * *} P<0.001$, by Poisson statistical analysis. (E) The percentage of hCD45+ cells in PB of secondary recipient mice was determined 4 months after transplantation (mean \pm SEM). $n=8-9$ mice per group. ${ }^{*} P<0.05$, by 2 -tailed Student's $t$ test. $(\mathbf{F}$ and $\mathbf{G})$ BM cells $\left(5 \times 10^{6}\right)$ from primary recipient mice transplanted with CD $34^{+} C D 133^{+} A D C R G 1^{+} C B$ cells or $C D 34{ }^{+} C D 133^{+} A D G R G 1-C B$ cells were infused into secondary recipient mice. The percentage of hCD45 ${ }^{+}$cells in BM was determined 4 months after transplantation (mean \pm SEM). $n=8-9$ mice per group. ${ }^{*} P<0.01$, by 2 -tailed Student's $t$ test. $(\mathbf{H}$ and $\mathbf{I})$ Flow cytometric plots showing the homing efficiency of

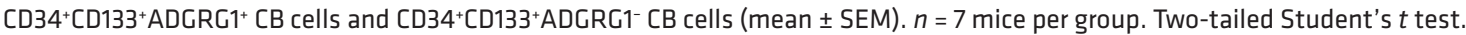


A

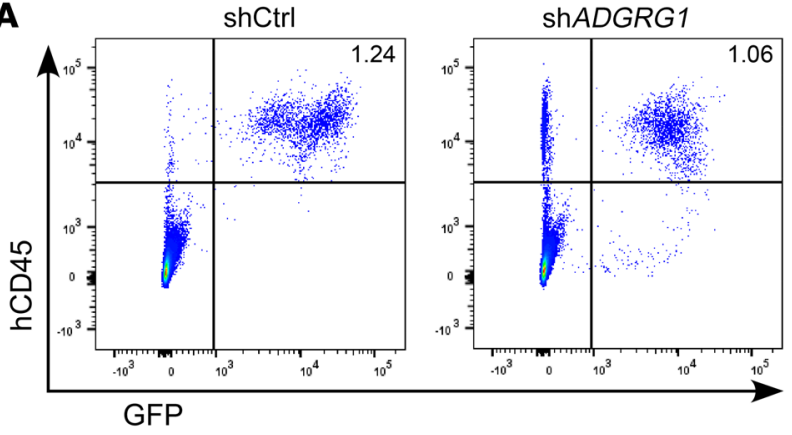

C

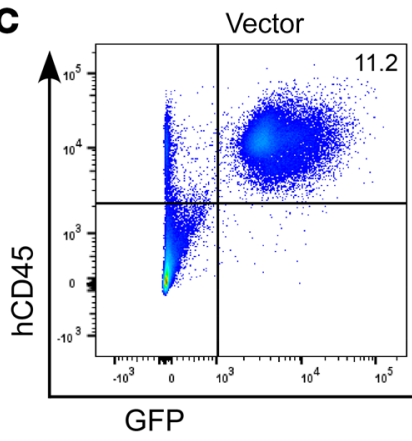

E

Enrichment plot: HSC_COMMOM_GENES

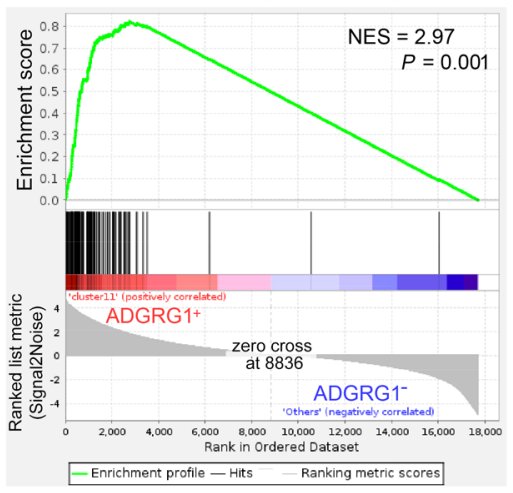

$A D G R G 1$

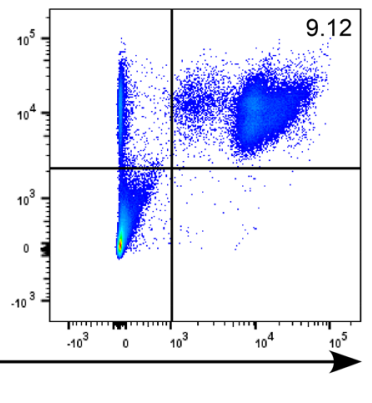

B

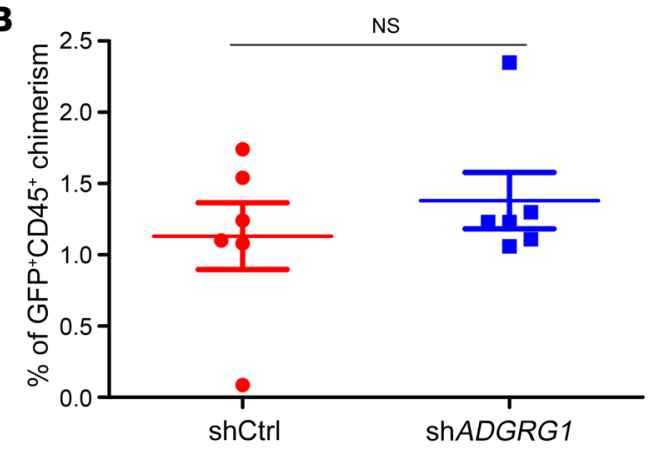

D

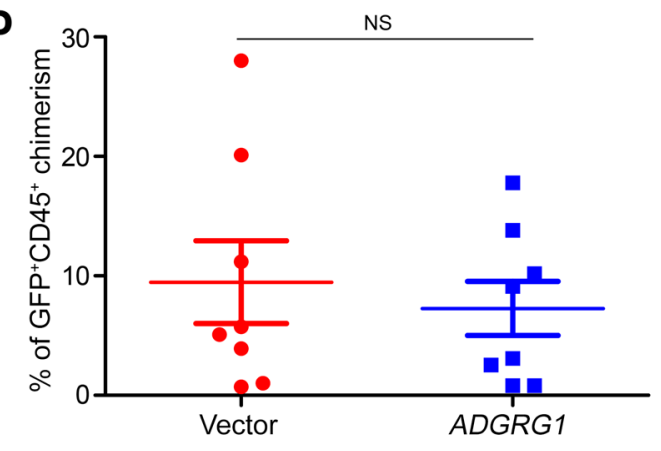

$\mathbf{F}$

Enrichment plot: HALLMARK_MYC_TARGETS_V1

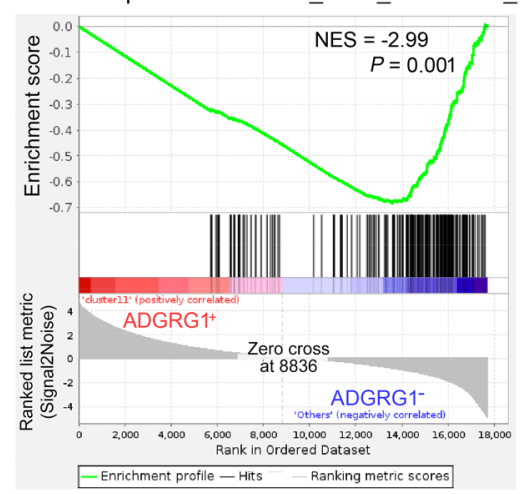

G

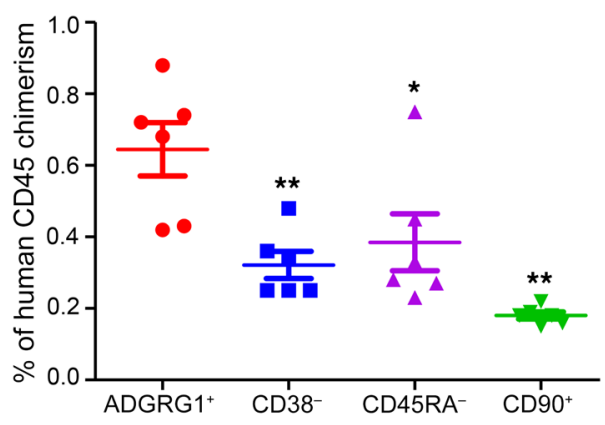

Figure 4. ADGRG1 enriches for HSC activity but is not involved in the regulation of self-renewal. (A) FACS plots showing GFP'hCD45 ${ }^{+}$chimerism from $B M$ of recipient mice transplanted with $\mathrm{CB} C D 34^{+}$cells infected by control (shCtrl) or shADGRG1 lentivirus 4 months after transplantation. (B) Quantification of GFP'hCD45+ chimerism from BM of recipient mice transplanted with CB CD34+ cells infected by shCtrl or shADGRG1 lentivirus 4 months after transplantation. $n=6$ mice per group. Data are shown as dot plots (mean \pm SEM). Two-tailed Student's $t$ test.(C) FACS plots showing GFP'hCD45 ${ }^{+}$chimerism from $\mathrm{BM}$ of recipient mice transplanted with $\mathrm{CB} C D 34^{+}$cells infected by vector or $A D G R G 1 \mathrm{OE}$ lentivirus. (D) Quantification of GFP+hCD45 chimerism from BM of recipients transplanted with CB CD34+ cells infected by shCtrl or shADGRG1 lentivirus 4 months after transplantation. $n=8$ mice per group. Data are shown as dot plots (mean \pm SEM). Two-tailed Student's $t$ test. (E) GSEA plots showing enrichment of HSC signature genes in ADCRC1+CD34+CD133 ${ }^{+}$CB cells (cluster 11 cells) after ex vivo culture. (F) GSEA plots showing the downregulation of MYC target genes in ADGRG1+CD34+CD133+ CB cells (cluster 11 cells) compared with ADGRG1-CD34+CD133+ CB cells. NES, normalized enrichment score. For E and F, Kolmogorov-Smirnov test was used. (C) Quantification of hCD45 chimerism from BM of recipients transplanted with ADCRG1+, CD38-, CD45RA', or CD90 cell populations sorted from CB CD34+ cells after ex vivo culturing (mean $\pm \mathrm{SEM}$ ). $n=6$ mice per group. ${ }^{*} P<0.05$ and ${ }^{* *} P<0.01$, by 1 -way ANOVA.

$\mathrm{CD} 34^{+} \mathrm{CD} 133^{+} \mathrm{ADGRG} 1^{+}$cells were bona fide HSCs and to assess the number of functional HSCs under oxidative stress upon ex vivo expansion. $\mathrm{CD} 34^{+} \mathrm{CD} 133^{+} \mathrm{ADGRG} 1^{+}$cells demonstrated significantly higher engraftment in both the bone marrow $(\mathrm{BM})$ and peripheral blood $(\mathrm{PB})$ of the primary recipient mice 4 months after transplantation compared with $\mathrm{CD} 34^{+} \mathrm{CD} 133^{+} \mathrm{ADGRG} 1^{-}$ cells (Figure 3, A and B, and Supplemental Figure 6, A-F). LDA and Poisson distribution analysis revealed an SRC frequency of 1:10,077 in CD34 ${ }^{+} \mathrm{CD} 133^{+} \mathrm{ADGRG}^{+}$cells and 1:135,972 in CD34 $4^{+}$
CD133 ${ }^{+}$ADGRG1 ${ }^{-}$cells, suggesting the presence of 99 SRCs and 7 SRCs (14.1-fold increase) in $1 \times 10^{6}$ ex vivo-cultured CD $34^{+} \mathrm{CD}_{133^{+}}$ cells (Figure 3, C and D, and Supplemental Tables 5 and 6). In order to assess whether ADGRG1 enriches for functional longterm HSCs with self-renewal capacity, we performed secondary transplantation experiments for another 4 months using BM cells from the primary recipient mice. The engraftment of human cell chimerism including both myeloid and lymphoid cells in BM and $\mathrm{PB}$ of the secondary recipients were significantly higher in $\mathrm{CD} 34^{+}$ 
$\mathrm{CD}_{133^{+}} \mathrm{ADGRG}^{+}$cells than in $\mathrm{CD} 34^{+} \mathrm{CD} 133^{+} \mathrm{ADGRG}^{-}$cells (Figure 3, E-G and Supplemental Figure 6, G and H). We also checked human cell reconstitution in the $\mathrm{PB}$ of both primary and secondary recipient mice at earlier time points. At week 8 after primary transplantation and at weeks 4 and 8 after secondary transplantation, the hCD 45 chimerism in PB was apparently higher in the CD $34^{+}$ $\mathrm{CD}_{133}{ }^{+} \mathrm{ADGRG} 1^{+}$group than in the $\mathrm{CD} 34^{+} \mathrm{CD} 133^{+} \mathrm{ADGRG} 1^{-}$group (Supplemental Figure 6, I and J). Moreover, we did not detect any significant differences in homing efficiencies between $\mathrm{CD} 34^{+}$ $\mathrm{CD}_{133}{ }^{+} \mathrm{ADGRG} 1^{+}$and $\mathrm{CD} 34^{+} \mathrm{CD} 133^{+} \mathrm{ADGRG}^{-}$cells (Figure $3, \mathrm{H}$ and I). These data suggest that ADGRG1 enriched for functional HSCs with long-term repopulating and self-renewing activity in $\mathrm{CB} \mathrm{CD} 34^{+} \mathrm{CD} 133^{+}$cells under mitochondrial oxidative stress conditions upon ex vivo culturing.

ADGRG1 knockdown did not significantly affect the engraftment of $\mathrm{CB} \mathrm{CD}_{3} 4^{+}$HSCs or HPCs (Supplemental Figure 7A and Figure 4, A and B), suggesting that ADGRG1 is dispensable for human CB HSC self-renewal. The $\mathrm{GFP}^{+} \mathrm{hCD} 45^{+}$cell chimerism in the $\mathrm{BM}$ was comparable in the ADGRG1-overexpressing (ADGRG1-OE) and vector control groups 4 months after transplantation (Figure 4, C and D), as was the $\mathrm{hCD}^{+} 3^{+}$myeloid and hCD19+ lymphoid chimerism (Supplemental Figure 7, B and C), suggesting that overexpression of ADGRG1 had no significant effect on the expansion or engraftment of CB HSCs.

GSEA indicated that in $\mathrm{CD} 34^{+} \mathrm{CD} 133^{+} \mathrm{ADGRG} 1^{+}$cells, a set of common human HSC genes (18) were highly enriched (Figure 4E), including MSI2 and MLLT3 (Supplemental Figure 7D), the overexpression of which promotes self-renewal of human HSCs $(15,16)$. MYC signaling-targeted genes, which are associated with HSC differentiation (19), were markedly downregulated in CD34 ${ }^{+}$ $\mathrm{CD}_{133^{+}} \mathrm{ADGRG}^{+}$cells (Figure $4 \mathrm{~F}$ ). Markers for freshly isolated HSCs including CD38, CD45RA, CD90, and CD49f (20) were not identified in our scRNA-Seq analysis. FACS analysis showed that $\mathrm{ADGRG}^{+} \mathrm{CD}^{2} 4^{+}$cells were almost $90 \%$ CD38-, 50\% CD45RA $10 \% \mathrm{CD}^{+} 0^{+}$, and 2\% CD49f+ (Supplemental Figure 7, E and F). To evaluate the enrichment of HSC activity by ADGRG1, we performed an in vivo transplantation experiment with ADGRG1 $1^{+}$

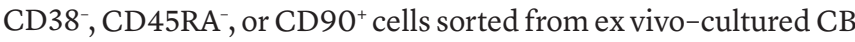
$\mathrm{CD} 4^{+}$cells. The engraftment capacity of ADGRG1 $1^{+}$cells was significantly higher than that observed in any of the other cell groups (Figure 4G). Thus, ADGRG1 enriched for a population of functional HSCs that highly expressed stemness-related genes under ex vivo culture stress conditions.

In steady state, the identity of freshly isolated human HSCs has been relatively well established $(1,20)$. However, upon ex vivo culturing, phenotypic HSCs labeled by these markers may not necessarily have the capacity to regenerate the blood system in vivo (10). Our results revealed that the engraftment capacity of cultured $\mathrm{CB} \mathrm{CD} 34^{+}$cells negatively correlated with cellular mitoROS levels. Incorporating scRNA-Seq and the in vivo transplantation experiment, we identified $\mathrm{CB} \mathrm{CD} 34^{+} \mathrm{CD} 133^{+} \mathrm{ADGRG} 1^{+}$cells as functional long-term repopulating cell populations under mitochondrial oxidative stress conditions induced upon ex vivo culturing, and demonstrated that many potential stemness regulators were enriched in this cell population. A small-molecule compound screen may be performed to find agonists of functional HSCs by targeting $\mathrm{CD} 34^{+} \mathrm{CD} 133^{+} \mathrm{ADGRG} 1^{+}$cells. Taken together, our study may improve the understanding of the regulation of human HSC maintenance and provide an alternative means for drug screening of agonists of HSC expansion.

\section{Methods}

Detailed methods are described in the Supplemental Methods.

Data availability. Bulk RNA-Seq data and scRNA-Seq data were deposited in the NCBI's Gene Expression Omnibus (GEO) database (GEO GSE179928 and GSE179361, respectively).

Study approval. All animal procedures were approved by the IACUC of Shanghai Jiao Tong University School of Medicine.

\section{Author contributions}

$\mathrm{BG}$ and HEB conceived the research, designed and supervised the experiments, interpreted data, and wrote the manuscript. JW supervised the data analysis and wrote the manuscript. QM and YT designed and supervised the experiments. YC designed the experiments, performed LDA, analyzed data, and wrote the manuscript. SF performed scRNA-Seq data analysis and wrote the manuscript. QD and RJ designed the experiments, collected CB samples, and prepared samples for transcriptomic analysis. JH, QW, and YJ and isolated stem cells and wrote the manuscript. SL performed bulk RNA-Seq data analysis. XH, MLC, and TT performed i.v. injections and interpreted data.

\section{Acknowledgments}

This work was supported by the National Key R\&D Program of China (2019YFA0111800); the Major Research Plan of National Natural Science Foundation of China (91957107); the General Program of the National Natural Science Foundation of China (81970095); the Shanghai Eastern Scholar Program (TP2018047, to BG); by US Public Health Service Grants from the NIH (Outstanding Investigator Awards R35 HL139599, U54 DK106846, to HEB); International Cooperation in Key R\&D Projects of Shanxi Province (201903D421026, to JFH and BG); and by a grant from the Innovative Research Team of High Level Local Universities in Shanghai (SSMU-ZDCX20180800).

Address correspondence to: Bin Guo, Department of Pathophysiology, Key Laboratory of Cell Differentiation and Apoptosis of Chinese Ministry of Education, Shanghai Jiao Tong University School of Medicine, 280, Chong-Qing South Road, West Bldg \#2, Shanghai 200025, China. Phone: 86.021.63846590.776936; Email: jybinguo@shsmu.edu.cn. Or to: Hal E. Broxmeyer, Department of Microbiology and Immunology, Indiana University School of Medicine, 950 West Walnut Street, R2-302, Indianapolis, Indiana 46202-5181, USA; Phone:1.317.274.7510; Email: hbroxmey@iupui. edu. Or to: Jun Wan, Department of Medical and Molecular Genetics, Indiana University School of Medicine, 410 W. 10th St., HITS 5013, Indianapolis, Indiana 46202, USA. Phone: 1.317.278.6445; Email: junwan@iu.edu. Or to: Qingyou Meng, Department of Vascular Surgery; General Surgery Clinical Center, Shanghai General Hospital, Shanghai 200080, China. Phone: 86.021.63240825; Email: qingyou.meng@shgh.cn. Or to: Yincheng Teng, Department of Obstetrics and Gynecology, Affiliated Sixth People's Hospital Shanghai Jiao Tong University, Shanghai 200233, China. Phone: 86.021.24058319; Email: ycteng@sjtu.edu.cn. 
1. Doulatov S, et al. Hematopoiesis: a human perspective. Cell Stem Cell. 2012;10(2):120-136.

2. Copelan EA. Hematopoietic stem-cell transplantation. N Engl J Med. 2006;354(17):1813-1826.

3. Morgan RA, et al. Hematopoietic stem cell gene therapy: progress and lessons learned. Cell Stem Cell. 2017;21(5):574-590.

4. Huang $X$, et al. Past, present, and future efforts to enhance the efficacy of cord blood hematopoietic cell transplantation. F100ORes. 2019;8:F1000 Faculty Rev-1833.

5. Fares I, et al. Cord blood expansion. Pyrimidoindole derivatives are agonists of human hematopoietic stem cell self-renewal. Science. 2014;345(6203):1509-1512.

6. Boitano AE, et al. Aryl hydrocarbon receptor antagonists promote the expansion of human hematopoietic stem cells. Science. 2010;329(5997):1345-1348.

7. Wagner JE Jr, et al. Phase I/II trial of StemRegenin-1 Expanded umbilical cord blood hematopoietic stem cells supports testing as a standalone graft. Cell Stem Cell. 2016;18(1):144-155.

8. Guo B, et al. Enhancing human cord blood hema- topoietic stem cell engraftment by targeting nuclear hormone receptors. Curr Opin Hematol. 2018;25(4):245-252.

9. Cohen S, et al. Hematopoietic stem cell transplantation using single UM171-expanded cord blood: a single-arm, phase 1-2 safety and feasibility study. Lancet Haematol. 2020;7(2):e134-e145.

10. Chen Y, et al. Phorbol ester induced ex vivo expansion of rigorously-defined phenotypic but not functional human cord blood hematopoietic stem cells: a cautionary tale demonstrating that phenotype does not always recapitulate stem cell function. Leukemia. 2019;33(12):2962-2966.

11. Pijuan-Sala B, et al. A single-cell molecular map of mouse gastrulation and early organogenesis. Nature. 2019;566(7745):490-495.

12. Zhou F, et al. Tracing haematopoietic stem cell formation at single-cell resolution. Nature. 2016;533(7604):487-492.

13. Chute JP, et al. Inhibition of aldehyde dehydrogenase and retinoid signaling induces the expansion of human hematopoietic stem cells. Proc Natl Acad Sci U S A. 2006;103(31):11707-11712.
14. Ghiaur G, et al. Regulation of human hematopoietic stem cell self-renewal by the microenvironment's control of retinoic acid signaling. Proc Natl Acad Sci U S A. 2013;110(40):16121-16126.

15. Calvanese V, et al. MLLT3 governs human haematopoietic stem-cell self-renewal and engraftment. Nature. 2019;576(7786):281-286.

16. Rentas S, et al. Musashi-2 attenuates AHR signalling to expand human haematopoietic stem cells. Nature. 2016;532(7600):508-511.

17. Jung $\mathrm{H}$, et al. TXNIP maintains the hematopoietic cell pool by switching the function of $\mathrm{p} 53$ under oxidative stress. Cell Metab. 2013;18(1):75-85.

18. Fares I, et al. EPCR expression marks UM171expanded $\mathrm{CD} 34^{+}$cord blood stem cells. Blood 2017;129(25):3344-3351.

19. Wilson A, et al. c-Myc controls the balance between hematopoietic stem cell selfrenewal and differentiation. Genes Dev. 2004;18(22):2747-2763.

20. Notta F, et al. Isolation of single human hematopoietic stem cells capable of longterm multilineage engraftment. Science. 2011;333(6039):218-221. 